of the positive charge of the membrane. Thus it seems likely that the positive charge attributable to the increase in the relative amount of $L P G$ in the mem. branes of cells grown at low $p H$ plays a part in inhibiting the entry of $\mathrm{H}^{+}$ions into the cell. It is not clear, however, what advantage accrues to the cell from the concomitant increase in nonelectrolyte permeability.

\section{DEVELOPMENTAL BIOLOGY Cell Cycles}

from a Correspondent

THE cell cycle in development and cell differentiation was the subject of the twenty-fourth meeting of the British Society for Developmental Biology at the University of Bristol on July 25-27. Professor J. Mitchison (University of Edinburgh) said in his opening address that the cell cycle, in showing morphogenesis and structural and chemical differentiation, mimics many of the processes that determine the development of higher organisms. Dr G. Steel (Institute for Cancer Research) then critically reviewed the methods used for determining the intermitotic period, and warned of the difficulties inherent in analyses of the cell cycle in complex developing systems.

In a group of papers on unicellular and acellular organisms, Dr M. Ord (University of Southampton) described how he has combined Amoeba nuclei and cytoplasms from different stages of the cell cycle, and Dr D. Ammermann (University of Tübingen) discussed the cell cycle and differentiation in micronuclei and macronuclei of the ciliate, Stylonychia. Dr P. John (Queen's University, Belfast) reviewed the work of the Belfast group on Chlorella, stressing the pattern of synthesis of several enzymes and the formation of mitochondria and cell wall material in relation to the cell cycle as a whole. The advantages of working with a plasmodial slime mould, in which large numbers of nuclei can achieve a high degree of natural synchrony, were described by Dr W. Grant (University of Leicester).

The higher plants were also well represented in the symposium. Dr M. Bennett (Plant Breeding Institute, Cambridge) stressed the importance of nucleotypic control and described how the mass of DNA in the nucleus is a significant factor in determining the duration of meiosis. Dr P. Barlow (ARC Developmental Botany Unit, Cambridge) was concerned with the factors which determine the pattern of mitosis in root meristems, including the relation between the cap and the cells of the quiescent centre. Dr R. Lyndon (University of Edinburgh) discussed the relationship between changes in the lengths of cell cycle phases and morphogenesis in the shoot apex, and Dr M. Yeoman (University of Edinburgh) described changes in enzymatic activities during the cell cycle in Jerusalem artichoke cells in vitro

A further group of contributions dealt with the cell cycle during early development. Professor G. Giudice (University of Palermo) discussed the significance of the slow maturation of ribosomal RNA and the synthesis of histones during sea urchin oogenesis, in relation to the modified cell cycle which is characteristic of the early stages of development of many embryos. An account of the beautifully regulated chronology of cell division during the early development of molluscs was given by Dr J. van den Biggelaar (Utrecht), who described how the duration of the cycle relates not only to the subsequent fate of the cells but also to their previous history.

Dr L. Hamilton (Middlesex Hospital, London) demonstrated how the natural synchrony of the initial cleavage stages of development can be used for analysing the sensitivity of the various parts of the cell cycle to irradiation. Dr P. Chibon (University of Grenoble) showed that, whereas the lengths of the $S, G 2$ and $M$ phases in the larval tissues of Pleurodeles shorten as the environmental temperature increases, the length of G1 increases. Dr G. Rudkin (University of Nijmegen) discussed the curtailed cell cycles of polytene cells of dipteran insects, in which chromosomal, nuclear and cell division are bypassed, leading to endoreduplication. $\mathrm{Dr} \mathrm{C}$. Graham (University of Oxford) said that the cell cycle is long during the early stages of cleavage in mammals, but shortens as cleavage proceeds while DNA accumulates by polyploidization in the trophoblast. $\mathrm{Dr}_{\mathrm{r}} \mathrm{M}$. Snow (University of Edinburgh) indicated that the cells of the inner cell mass are particularly susceptible to radiation damage (including damage by ${ }^{3} \mathrm{H}$ thymidine) at the point in cleavage where the cell cycle is shortening.

The final session dealt largely with the control of cell proliferation by extrinsic factors, Dr P. Harrison (Beatson Institute, Glasgow) showed that erythropoietin induces both proliferation in proerythroblasts and an increased rate of haemoglobinization in foetal liver cells in vitro, and $\mathrm{Dr} R$. Cole (University of Sussex) discussed genetic defects which upset the regulation of red cell formation in foetal mice. Dr D. Hardy (University of Birmingham) reviewed the mitotic activation of lymphocytes. He emphasized that there is not enough experimental evidence to support the idea that the process is the consequence of the direct action of stimulants, such as PHA, on the genome, evoking the action of new genes; it was far simpler to assume a rather less specific action at the cytoplasmic level.

\title{
Active Fragments from Growth Hormone
}

THERE have been several reports of the preparation of fragments of pituitary growth hormone which retain biological activity. An interesting example came from Chillemi and Pecile last year (Experientia, 27, 385; 1971) who synthesized two peptides with growth promoting activity. Some doubt was thrown on this work, however, by the subsequent discovery that the aminoacid sequence of human growth hormone, on which the authors had based their synthetic work, was incorrect. In next Wednesday's Nature New Biology (August 23) Chillemi, Pecile and Aiello report the synthesis of two new peptides, according to the revised sequence.

The sequence of human growth hormone (a protein of 190 amino-acid residues) includes four partially duplicated regions. Chillemi et al. prepared peptides corresponding to two of these regions (residues 87 to 123 and 124 to 155) by chemical synthesis using the solid phase method. Both peptides showed substantial biological activity when tested in a growth hormone biossay (the "tibia test"). The work suggests that intact growth hormone possesses at least two biologically active sites, and, as the authors point out, there may well be others.

The success of the synthetic approach of Chillemi et al. to the preparation of fragments of growth hormone is particularly important because it opens the way to the preparation of peptides corresponding to many different regions of growth hormone, as well as analogues of them. This should enable detailed structure/function relationships to be carried out with this hormone. Ideally future work should in clude a full characterization of the biological properties of the fragments as well as synthesis of further peptides.

The observation that peptides corresponding to quite small regions of growth hormone may retain biological activity suggests that such fragments may play a physiological part in mediating the actions of growth hormone. (Such a hypothesis was proposed for the diabetogenic actions of growth hormone by Bornstein.) Peptides with growth promoting activity might also be suitable for clinical use-and help relieve the current shortage of human growth hormone. 